\title{
СРАВНИТЕЛЬНЫЙ АНАЛИЗ КАНОНИЧЕСКИХ ФОРМ МНОГОМЕРНЫХ ЛИНЕЙНЫХ СИСТЕМ
}

\author{
(Представил Н. Алумяэ)
}

\section{1. Введение}

Рассмотрим линейную многомерную систему

$$
\begin{aligned}
& \dot{x}(t)=A x(t)+B u(t), \\
& y(t)=C x(t),
\end{aligned}
$$

где $u(t), x(t)$ и $y(t)$ - векторы входа, состояния и выхода размерности $m, n$ и $p$ соответственно, а $A, B$ и $C$ - матрицы подходящей размерности.

Сложность идентификации и моделирования динамического объекта, а также синтеза системы управления во многом зависит от количества параметров объекта. В общем случае тройка матриц $(A, B, C)$ включает в себя $K=n(m+n+p)$ параметров. Ввиду инвариантности отображения вход-выход относительно линейного преобразования на пространстве состояний существует множество эквивалентных представлений системы (1), т. е. матрицы $A, B$ и $C$ могут быть заменены матрицами $\bar{A}, \bar{B}$ и $\bar{C}$, найденными по формулам

$$
\bar{A}=T A T^{-1}, \quad \bar{B}=T B, \quad \bar{C}=C T^{-1},
$$

где $T$ - любая невырожденная матрица порядка $n \times n$. Наибольший интерес вызывают канонические формы (к. ф.) модели состояния (1), в которых определенное число элементов матриц $\bar{A}, \bar{B}$ и $\bar{C}$ строго фиксировано (0 и 1$)$.

\section{2. Классификация канонических форм}

Благодаря соотношениям эквивалентности (2) существует много к. ф. модели состояния (1) $\left[{ }^{1-6}\right]$. Их можно классифицировать по различным признакам.

При изучении к. ф. важное значение имеет понятие дуальных систем. Система $(\widetilde{A}, \widetilde{B}, \widetilde{C})$ называется дуальной к системе $(A, B, C)$, если

$$
\tilde{A}=A^{\prime}, \quad \widetilde{B}=C^{\prime}, \quad \widetilde{C}=B^{\prime},
$$

где $A^{\prime}$ обозначает транспонированную матрицу $A$. Нетрудно видеть, что существуют пары дуальных к. ф. с симметричной структурой матриц $\bar{B}$ и $\bar{C}$, 
Классификация канонических форм многомерных линейных систем

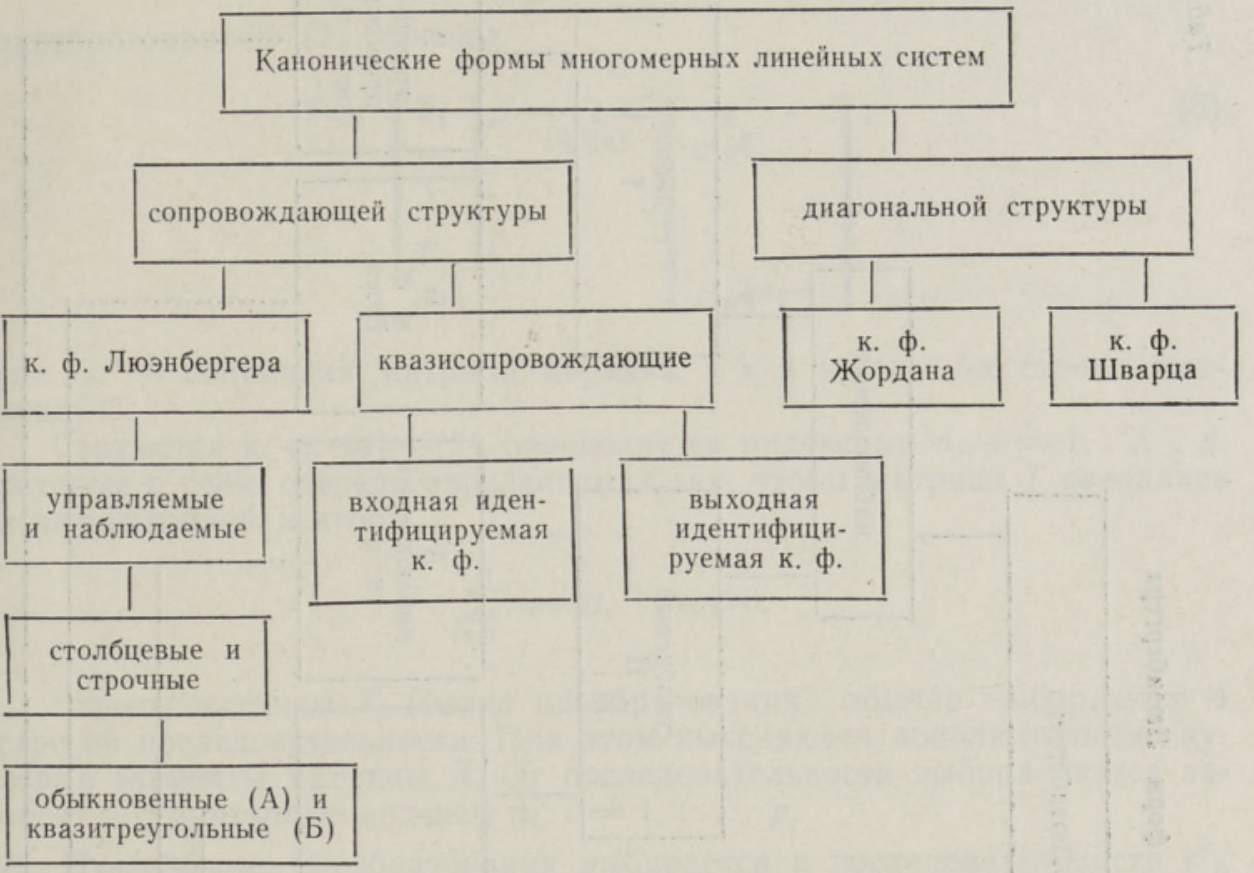

В тройке матриц $(A, B, C)$ центральную роль играет матрица $A$, так как ею определяются динамические свойства системы. Поэтому в к. ф. матрица $\bar{A}$ имеет, как правило, простую структуру. По структуре матрицы $\bar{A}$ к. ф. можно разбить на две группы: к. ф. диагональной структуры (к. ф. Жордана, к. ф. Шварца) и к. ф. сопровождающей структуры (к. ф. Люэнбергера, к. ф. Будина и др.). Можно отметить еще квазисопровождающую структуру (блочная структура матрицы $\bar{A}$ подобна сопровождающей) и структуру с диагональными сопровождающими блоками (к. ф. Люэнбергера), а также столбцевую и строчную сопровождающие структуры (дуальные канонические формы). При более подробном изучении к. ф. Люэнберғера можно выделить еще квазитреугольную структуру матрицы $\bar{A}$ (к. ф. Бюси).

Классификация к. ф. многомерных линейных систем представлена в табл. 1, а к. ф. Люэнбергера - в табл. 2.

В дальнейшем будем рассматривать лишь полностью наблюдаемую пару матриц $(A, C)$, так как матрица $B$ не включает в себя фиксированных элементов. Дуальным подходом можно получить аналогичные результаты и для полностью управляемой пары $(A, B)$.

\section{3. Первая каноническая форма Люэнбергера}

Пусть система (1) минимально наблюдаема, т. е. для достижения полной наблюдаемости системы необходимы все $p$ выходов. Тогда система (1) приводится к первой канонической форме Люэнбергера [ $\left.{ }^{4}\right]$

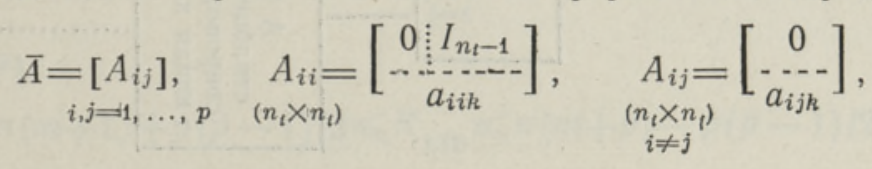


ปั

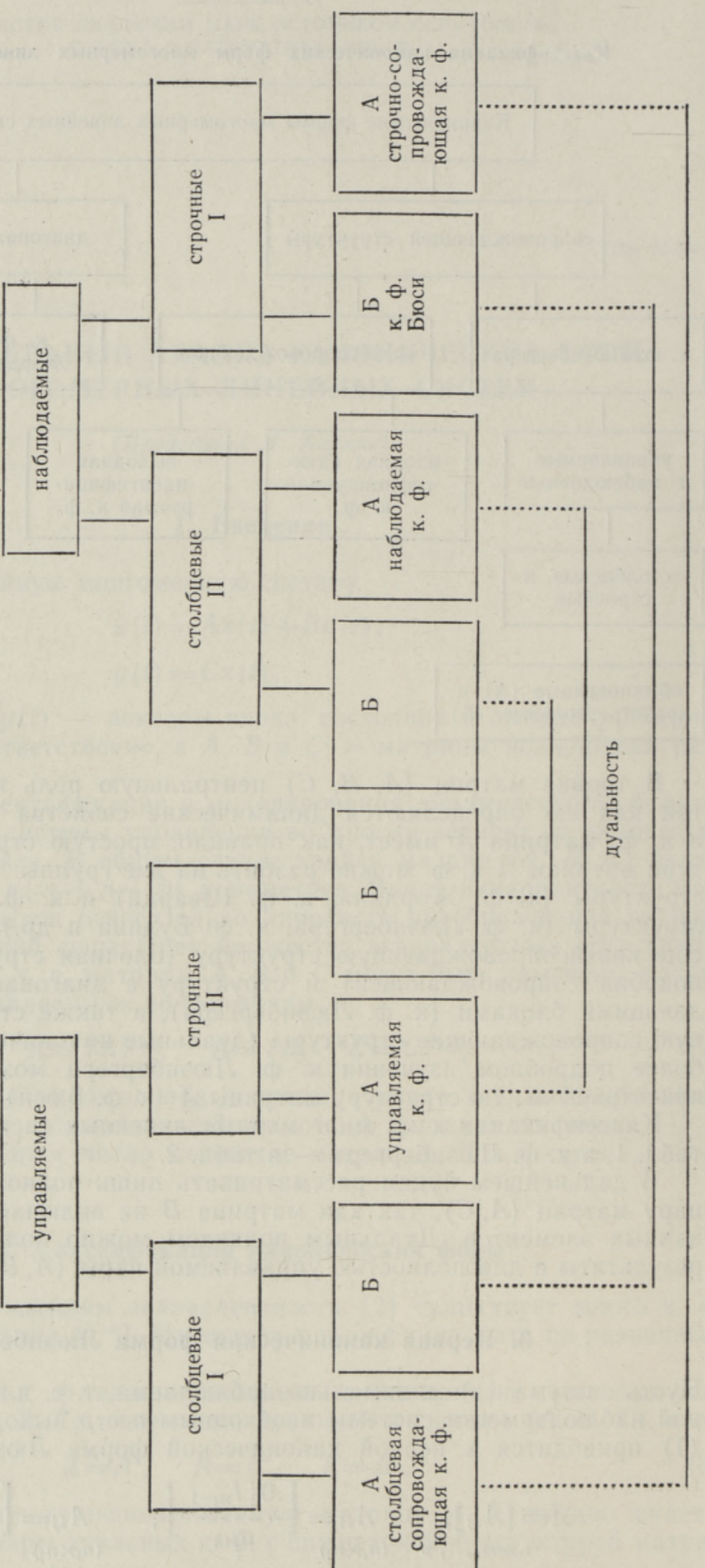




$$
\bar{C}=\left[\begin{array}{lll}
c^{\prime}{ }_{i j}
\end{array}\right], \quad \underset{\left(1 \times n_{t}\right)}{c_{i i}^{\prime}}=\left[\begin{array}{llll}
1 & 0 & \ldots & 0
\end{array}\right], \quad \underset{\left(1 \times n_{t}\right)}{c^{\prime}}{ }_{i j}=0
$$

преобразованием (2), причем

$$
T=\left[\begin{array}{c}
T_{1} \\
\cdot \\
\cdot \\
\dot{T}_{p}
\end{array}\right], \quad \begin{gathered}
T_{i}= \\
\left(n_{t} \times n\right)
\end{gathered}\left[\begin{array}{l}
c^{\prime}{ }_{i} \\
c^{\prime}{ }_{i} A \\
\cdot \\
\cdot \\
\dot{c}^{\prime}{ }_{i} A^{n_{t}-1}
\end{array}\right],
$$

где $I_{n}$ - единичная матрица порядка $n \times n$ и $c^{\prime}{ }_{i}-i$-я строка матрицы $C$.

Структура к. ф. (4)-(5) определяется индексами $n_{i}, i=1, \ldots, p$, которые в свою очередь определягтся так, чтобы матрица $T$ оказалась невырожденной и чтобы

$$
\sum_{i=1}^{p} n_{i}=n, \quad n_{i} \neq 0
$$

Строки матрицы $T$ (базис преобразования) обычно выбираются в строгой последовательности. При этом выясняются дополнительные нулевые элементы матрицы $\bar{A}$. От последовательности выбора базиса зависят и структурные индексы $n_{i}, i=1, \ldots, p$.

А. Пусть базис преобразования выбирается в последовательности $c^{\prime}{ }_{1}$, $c^{\prime}{ }_{2}, \ldots, c_{p}^{\prime}, c^{\prime}{ }_{1} A, c^{\prime}{ }_{2} A, \ldots$. Тогда получим строчно-сопровождающую к. ф. $\left[{ }^{6}\right]$, причем $\left[{ }^{7}\right]$

$$
\begin{aligned}
& a_{i j k}=0, \text { если } i<j, \quad k>n_{i}, \\
& i>j, \quad k>n_{i}+1 \text {. }
\end{aligned}
$$

Количество параметров строчно-сопровождающей к. ф. определяется формулой $\left[{ }^{8}\right]$

$$
K_{L \mathrm{IA}}=n(m+p)-\sum_{i=1}^{p}\left[\sum_{\substack{j=1 \\ n_{l}>n_{l}}}^{i-1}\left(n_{i}-n_{j}\right)+\sum_{\substack{j=i+1 \\ n_{l}>n_{l}}}^{p}\left(n_{i}-n_{j}-1\right)\right],
$$

причем

$$
n(m+1)+p(p-1) \leqslant K_{L I A} \leqslant n(m+p) .
$$

Б. Пусть базис преобразования выбирается в последовательности $c^{\prime}{ }_{1}, c^{\prime}{ }_{1} A, \ldots c^{\prime}{ }_{1} A^{n_{1}-1}, c^{\prime}{ }_{2}, c^{\prime}{ }_{2} A, \ldots$. Тогда получим к. ф. Бюси $\left[{ }^{1}\right]$, причем

$$
a_{i j k}=0 \text {, если } i<j .
$$

Количество параметров к. ф. Бюси определяется формулой [8 $\left.{ }^{8}\right]$

$$
K_{L I \mathrm{~S}}=n m+\sum_{j=1}^{p}(p-j+1) n_{j}
$$

причем $\left[{ }^{9}\right]$

$$
n(m+1)+p(p-1) / 2 \leqslant K_{L I Б} \leqslant n(m+p)-p(p-1) / 2 .
$$




\section{4. Вторая каноническая форма Люэнбергера}

Система (1) приводится ко второй к. ф. Люэнбергера [ $\left.{ }^{4}\right]$

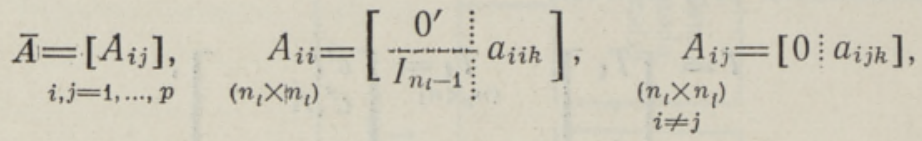

$$
\begin{aligned}
& \bar{C}=\left[c^{\prime}{ }_{i j}\right]
\end{aligned}
$$

преобразованием (2), если

$$
\begin{aligned}
T & =\left[T_{1} \ldots T_{p}\right], \\
\underset{\left(n \times n_{f}\right)}{T_{j}} & {\left[v_{j}, A v_{j}, \ldots, A^{n_{j}-1} v_{j}\right], }
\end{aligned}
$$

причем

$$
c^{\prime}{ }_{i} A^{k-1} v_{j}=\left\{\begin{array}{lll}
1, & \text { если } & k=n_{i}, \quad i=j, \\
0, & \text { если } & k<n_{i} .
\end{array}\right.
$$

А. Пусть базис преобразования выбирается в последовательности $c^{\prime}{ }_{1}, c^{\prime}{ }_{2}, \ldots, c^{\prime}{ }_{p}, c^{\prime}{ }_{1} A, c_{2}^{\prime} A, \ldots$. Тогда получим т. н. наблюдаемую к. ф. [6]. Можно показать, что

$$
\begin{aligned}
& a_{i j k}=0, \quad \text { если } \quad k>n_{j} ; \\
& c_{i j k}=\left\{\begin{array}{lll}
1, & \text { если } & k=n_{i}, \quad i=j, \\
0, & \text { если } & k<n_{j}, \\
& k=n_{j}, \quad j>i, \\
& k=n_{j}, \quad j<i, \quad n_{i}<n_{j} .
\end{array}\right.
\end{aligned}
$$

Количество параметров определяется формулой

$$
K_{L I I A}=n(m+p)-\sum_{i=1}^{p-1} \sum_{j=i+1}^{p}\left[\left|n_{i}-n_{j}\right|-\operatorname{sign}\left(n_{i}-n_{j}+\left|n_{i}-n_{j}\right|\right)\right],
$$

причем

$$
n(m+1)+(p-1) p \leqslant K_{L I \mathrm{AA}} \leqslant n(m+p) .
$$

Б. Пусть базис преобразования выбирается в последовательности $c^{\prime}{ }_{1}, c^{\prime}{ }_{1} A, \ldots, c^{\prime}{ }_{1} A^{n_{1}-1}, c^{\prime}{ }_{2}, c^{\prime}{ }_{2} A, \ldots$. Можно показать, что тогда

$$
\begin{aligned}
& a_{i j k}=0, \\
& c_{i j k}=\left\{\begin{array}{lll}
1, & \text { если } & j>i, \\
0, & \text { если } & j<i, k>n_{j} .
\end{array}\right.
\end{aligned}
$$

Количество параметров

$$
K_{L \text { Іь }}=n m+\sum_{i=1}^{p}\left[\sum_{j=i}^{p} n_{j}+\sum_{j=1}^{i-1}\left(n_{j}-n_{i}\right)\right]
$$


причем

$$
n(m+1)+p(p-1) / 2 \leqslant K_{L \text { IІБ }} \leqslant n(m+p)-p(p-1) / 2 .
$$

\section{5. Каноническая форма Будина}

Пусть ранг $C=p$. Тогда система (1) приводится к канонической форме Будина $\left[{ }^{2}\right]$

$$
\begin{aligned}
& \bar{A}=S\left[\begin{array}{c:c}
0 & I_{n-p} \\
\hdashline A^{*}
\end{array}\right], \underset{(p \times n)}{A^{*}}=\left[a_{i j}\right], \\
& \bar{C}=\left[\begin{array}{l}
I_{p} \vdots 0 \\
\hdashline 0
\end{array}\right.
\end{aligned}
$$

где $S$ - селектор-матрица порядка $n \times n$, преобразованием (2), если

$$
T=\left[\begin{array}{c}
T_{1} \\
\cdot \\
\dot{T}_{v}
\end{array}\right], \quad T_{k}=\left[\begin{array}{c}
c^{\prime}{ }_{k_{1}} A^{k-1} \\
\cdot \\
\vdots \\
c^{\prime}{ }_{k_{r_{k}}} A^{k-1}
\end{array}\right], \quad \begin{aligned}
& \\
& k_{i}<k_{i+1} \\
& r_{k} \leqslant p,
\end{aligned}
$$

где $v$ - индекс наблюдаемости, т. е. минимальное число такое, что ранг $\left[C^{\prime}, A^{\prime} C^{\prime}, \ldots,\left(A^{\prime}\right)^{v-1} C^{\prime}\right]=n$.

В матрице $\bar{A}$ можно зафиксировать еще дополнительные нулевые элементы [ $\left.{ }^{9}\right] a_{i j}=0$, если $j>\sum_{l=1}^{k} r_{l}, i \leqslant p-r_{k}$, а количество параметров к. ф. (15)-(16) удовлетворяет неравенствам

$$
n(m+1)+p(p-1) \leqslant K_{B} \leqslant n(m+p) .
$$

Особенно удобную структуру принимает к. ф. Будина, если $S=I_{n}$ $\left[{ }^{10}\right]$. Тогда получается т. н. выходная идентифицируемая к. ф. $\left[{ }^{6}\right]$.

\section{6. Каноническая форма Жордана}

Пусть элементы матриц $(A, B, C)$ комплексные числа. Тогда матрица $A$ : приводится преобразованием (2) к жордановой нормальной форме [ $\left.{ }^{11}\right]$

$$
\begin{aligned}
& \bar{A}=\operatorname{diag} A_{i}, \quad i=1, \ldots, k, \\
& A_{i}=\operatorname{diag} A_{i j}, \quad j=1, \ldots, q_{i} \text {, }
\end{aligned}
$$

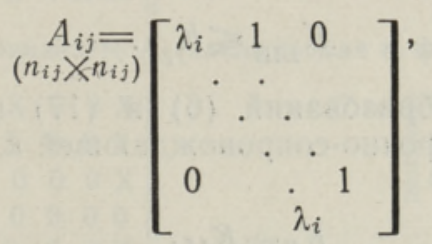

где $\lambda_{i}-$ собственное значение матрицы $A, k-$ число различных собственных значений, $q_{i}$ - количество жордановых блоков, связанных с $\lambda_{i}$. Разобьем матрицу $\bar{C}$ на $\left(p \times n_{i j}\right)$-блоки

$$
\bar{C}=\left[C_{11} \ldots C_{1 q_{1}} \ldots C_{k q_{k}}\right],
$$




$$
\begin{aligned}
& C_{i j}=\left[c_{r s}(i j)\right], \quad r=1, \ldots, p, \\
& s=1, \ldots, n_{i j} \text {. }
\end{aligned}
$$

При подходящей нумерации выходов можно зафиксировать следующие элементы в матрице $\bar{C}$ :

$$
c_{r s}(i j)=\left\{\begin{array}{lll}
1, & \text { если } & s=1, r=j, \\
0, & \text { если } & s=1, r<j, \\
& s>n_{i j}-n_{i r}, 1 \leqslant r \leqslant q_{i} .
\end{array}\right.
$$

Количество параметров к. ф. Жордана равняется [ $\left.{ }^{3}\right]$

$$
K_{J}=n(m+p)+k-\sum_{i=1}^{k} \sum_{j=1}^{q_{t}}(2 j-1) n_{i j}
$$

причем

$$
n m+1 \leqslant K_{J} \leqslant n(m+p) .
$$

\section{7. Сравнение канонических форм}

Постараемся сравнить количество параметров рассмотренных к. ф. Сравнение по формулам (7), (9), (11), (13), (19) затрудняется тем, что индексы, определяющие структуру к. ф. (единичные элементы селектор-матриц, структурные индексы, размеры жордановых блоков), различны и зависимость между ними неизвестна. В общем случае можно показать, что

$$
\begin{aligned}
& K_{\text {LIA }}=K_{L \text { IIA }} \\
& K_{\text {LIБ }}=K_{L \text { IIБ }} .
\end{aligned}
$$

При этом нетрудно заметить, что

$$
\begin{aligned}
& K_{A}^{L I I} \leqslant K_{A}^{L I}, \\
& K_{C}^{L I I} \geqslant K_{C}^{L I}=0,
\end{aligned}
$$

где $K_{\boldsymbol{A}}$ и $K_{C}$ обозначают количество параметров матриц $\bar{A}$ и $\bar{C}$ соответственно. Отметим еще, что различные разновидности к. ф. Люэнбергера имеют различные структурные индексы $n_{i}$, причем выполняются следующие неравенства

$$
n_{i \max }^{L \mathrm{~B}} \geqslant n_{i \max }^{L} \geqslant n_{i \max }^{L A}=v .
$$

При подходящей нумерации выходов к. ф. Бюси имеет минимальное число параметров среди к. ф. Люэнбергера $\left[{ }^{8}\right]$ :

$$
K_{L \text { IБ }} \leqslant K_{L I} \text {. }
$$

Сравнение матриц преобразований (6) и (17) показывает, что к. ф. Будина получается из строчно-сопровождающей к. ф. перенумерованием состояний. Значит,

$$
K_{B}=K_{L I A} .
$$

Сравнение границ возможных изменений числа параметров (8), (10), (12), (14), (18), (20) позволяет установить следующие неравенства:

$$
K_{\min }^{J} \leqslant K_{\min }^{L I I \mathrm{II}}=K_{\min }^{L \mathrm{IL}} \leqslant K_{\min }^{L I \mathrm{II}}=K_{\min }^{L I \mathrm{~A}}=K_{\min }^{B},
$$




$$
K_{\max }^{L I Б}=K_{\max }^{L I L} \leqslant K_{\max }^{L I A}=K_{\max }^{L I I A}=K_{\max }^{B}=K_{\max }^{J},
$$

где $K_{\min }$ и $K_{\max }$ обозначают нижнюю и верхнюю границы числа параметров. Надо отметить, что нижняя граница достигается при довольно редких обстоятельствах. Так, например, $K_{\min }{ }^{J}$ достигается, если матрица $A$ имеет лишь одно собственное число $\lambda$ кратности $n$, а $K_{\min }{ }^{L}-$ тогда, когда из первых $p-1$ выходов наблюдаемы лишь одномерные подсистемы. Зато верхняя граница получается довольно часто (циклические и «generic» системы).

Из сказанного следует, что по количеству параметров некоторые преимущества имеют разновидности ІБ и ІІБ к. ф. Люэнбергера для минимально наблюдаемых систем. В основном применение той или иной к. ф. определяется ее структурой. Так, например, первая к. ф. Люэнбергера и к. ф. Будина подходят для идентификации системы $\left[{ }^{12}\right]$. В частности, строчно-сопровождающая к. ф. подходит для решения задачи частичной реализации $\left[{ }^{13}\right]$. Наблюдаемая к. ф. подходит для синтеза наблюдателя (идентификатора состояния) [14], а управляемая к. ф. - для синтеза регулятора $\left[{ }^{4}\right]$. К. ф. Жордана найдет широкое применение в задачах модального управления, а также для качественного анализа линейных многомерных систем [15].

Прим ер. Пусть $p=3, n=9$ и структурные индексы $n_{1}=4, n_{2}=2, n_{3}=3$. Төгда матрицы $A$ и $C$ имеют следующую структуру (x- неопеделенные параметры): I к. ф. Люэнбергера, разновидность А (строчно-сопровождающая к. ф.)

$$
A=\left\|\begin{array}{lllllllll}
0 & 1 & 0 & 0 & 0 & 0 & 0 & 0 & 0 \\
0 & 0 & 1 & 0 & 0 & 0 & 0 & 0 & 0 \\
0 & 0 & 0 & 1 & 0 & 0 & 0 & 0 & 0 \\
\mathrm{X} & \mathrm{X} & \mathrm{X} & \mathrm{X} & \mathrm{X} & \mathrm{X} & \mathrm{X} & \mathrm{X} & \mathrm{X} \\
0 & 0 & 0 & 0 & 0 & 1 & 0 & 0 & 0 \\
\mathrm{X} & \mathrm{X} & \mathrm{X} & 0 & \mathrm{X} & \mathrm{X} & \mathrm{X} & \mathrm{X} & 0 \\
0 & 0 & 0 & 0 & 0 & 0 & 0 & 1 & 0 \\
0 & 0 & 0 & 0 & 0 & 0 & 0 & 0 & 1 \\
\mathrm{X} & \mathrm{X} & \mathrm{X} & \mathrm{X} & \mathrm{X} & \mathrm{X} & \mathrm{X} & \mathrm{X} & \mathrm{X}
\end{array}\right\|, \quad C=\| \begin{array}{lll}
1 & 0 & 0 \\
0 & 0 & 0 \\
0 & 0 & 0
\end{array}
$$

I к. ф. Люэнбергера, разновидность Б (к. ф. Бюси)

$$
\begin{gathered}
A=\left\|\begin{array}{lllllllll}
0 & 1 & 0 & 0 & 0 & 0 & 0 & 0 & 0 \\
0 & 0 & 1 & 0 & 0 & 0 & 0 & 0 & 0 \\
0 & 0 & 0 & 1 & 0 & 0 & 0 & 0 & 0 \\
\mathrm{X} & \mathrm{X} & \mathrm{X} & \mathrm{X} & 0 & 0 & 0 & 0 & 0 \\
0 & 0 & 0 & 0 & 0 & 1 & 0 & 0 & 0 \\
\mathrm{X} & \mathrm{X} & \mathrm{X} & \mathrm{X} & \mathrm{X} & \mathrm{X} & 0 & 0 & 0 \\
0 & 0 & 0 & 0 & 0 & 0 & 0 & 1 & 0 \\
0 & 0 & 0 & 0 & 0 & 0 & 0 & 0 & 1 \\
\mathrm{X} & \mathrm{X} & \mathrm{X} & \mathrm{X} & \mathrm{X} & \mathrm{X} & \mathrm{X} & \mathrm{X} & \mathrm{X}
\end{array}\right\|, \quad C=\left\|\begin{array}{lllllllll}
1 & 0 & 0 & 0 & 0 & 0 & 0 & 0 & 0 \\
0 & 0 & 0 & 0 & 1 & 0 & 0 & 0 & 0 \\
0 & 0 & 0 & 0 & 0 & 0 & 1 & 0 & 0
\end{array}\right\|, \\
\\
\end{gathered}
$$

II к. ф. Люэнбергера, разновидность А (наблюдаемая к. ф.)

$$
A=\left\|\begin{array}{lllllllll}
0 & 0 & 0 & X & 0 & X & 0 & 0 & X \\
1 & 0 & 0 & X & 0 & X & 0 & 0 & X \\
0 & 1 & 0 & X & 0 & 0 & 0 & 0 & X \\
0 & 0 & 1 & X & 0 & 0 & 0 & 0 & 0 \\
0 & 0 & 0 & X & 0 & X & 0 & 0 & X \\
0 & 0 & 0 & X & 1 & X & 0 & 0 & X \\
0 & 0 & 0 & X & 0 & X & 0 & 0 & X \\
0 & 0 & 0 & X & 0 & X & 1 & 0 & X \\
0 & 0 & 0 & X & 0 & 0 & 0 & 1 & 0
\end{array}\right\|
$$$$
C=\left\|\begin{array}{lllllllll}
0 & 0 & 0 & 1 & 0 & 0 & 0 & 0 & 0 \\
0 & 0 & 0 & \mathrm{X} & 0 & 1 & 0 & 0 & 0 \\
0 & 0 & 0 & \mathrm{X} & 0 & 0 & 0 & 0 & 1
\end{array}\right\|,
$$$$
K_{A, c}=25 .
$$ 
II к. ф. Люэнбергера, разновидность Б.

$A=\left\|\begin{array}{lllllllll}0 & 0 & 0 & \mathrm{X} & 0 & 0 & 0 & 0 & 0 \\ 1 & 0 & 0 & \mathrm{X} & 0 & 0 & 0 & 0 & 0 \\ 0 & 1 & 0 & \mathrm{X} & 0 & 0 & 0 & 0 & 0 \\ 0 & 0 & 0 & \mathrm{X} & 0 & \mathrm{X} & 0 & 0 & 0 \\ 0 & 0 & 0 & \mathrm{X} & 1 & \mathrm{X} & 0 & 0 & 0 \\ 0 & 0 & 0 & \mathrm{X} & 0 & \mathrm{X} & 0 & 0 & \mathrm{X} \\ 0 & 0 & 0 & \mathrm{X} & 0 & \mathrm{X} & 1 & 0 & \mathrm{X} \\ 0 & 0 & 0 & \mathrm{X} & 0 & 0 & 0 & 1 & \mathrm{X}\end{array}\right\|, \quad C=\left\|\begin{array}{lllllllll}0 & 0 & 0 & 1 & 0 & 0 & 0 & 0 & 0 \\ 0 & 0 & \mathrm{X} & \mathrm{X} & 0 & 1 & 0 & 0 & 0 \\ 0 & 0 & 0 & \mathrm{X} & 0 & 0 & 0 & 0 & 1\end{array}\right\|$,

К. ф. Будина

$$
\begin{aligned}
& A=\left\|\begin{array}{lllllllll}
0 & 0 & 0 & 1 & 0 & 0 & 0 & 0 & 0 \\
0 & 0 & 0 & 0 & 1 & 0 & 0 & 0 & 0 \\
0 & 0 & 0 & 0 & 0 & 1 & 0 & 0 & 0 \\
0 & 0 & 0 & 0 & 0 & 0 & 1 & 0 & 0
\end{array}\right\|, \quad C=\left\|\begin{array}{lllllllll}
1 & 0 & 0 & 0 & 0 & 0 & 0 & 0 & 0 \\
0 & 1 & 0 & 0 & 0 & 0 & 0 & 0 & 0 \\
0 & 0 & 1 & 0 & 0 & 0 & 0 & 0 & 0
\end{array}\right\|, \\
& \begin{array}{lllllllll}
\mathrm{X} & \mathrm{X} & \mathrm{X} & \mathrm{X} & \mathrm{X} & \mathrm{X} & \mathrm{X} & 0 & 0 \\
0 & 0 & 0 & 0 & 0 & 0 & 0 & 1 & 0
\end{array} \\
& \begin{array}{lllllllll}
0 & 0 & 0 & 0 & 0 & 0 & 0 & 0 & 1
\end{array}
\end{aligned}
$$

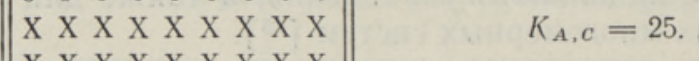

ЛИТЕРАТ У РА

1. Bucy, R. S., IEEE Trans. Automat. Contr., 13, № 5, 567-569 (1968).

2. B u d i n, M. A., IEEE Trans. Automat. Contr., 17, № 4, 554 (1972).

3. He y m a n n, M., Int. J. Contr., 12, № 6, 913-927 (1970).

4. Lue n berger, D. G., IEEE Trans. Automat. Contr., 12, № 3, 290-293 (1967).

5. Shi e h, L.-S., S a chet i, S., Int. J. Contr., 27, № 2, 245-259 (1978).

6. S in ha, N. K., Roz s a, P., Int. J. Contr., 23, № 6, 865-883 (1976).

7. Gupta, R. D., F a i rm a n, F. W., IEEE Trans. Automat. Contr., 19, № 4, 440-441 (1974).

8. Нургес Ю., Изв. АН ЭССР, Физ. Матем., 26, № 1, 56-63 (1977).

9. B u c y, R. S., A c k e r m a n n, J., Regelungstechnik, 18, № 10, 451-452 (1970).

10. Нургес Ю., Я аксоо Ю., Изв. АН ЭССР, Физ. Матем., 24, № 3, 270-276 (1975).

11. Г а н т м а х е р Ф. Р., Теория матриц, М., Физматгиз, 1967.

12. Guidorzi, R., Automatica, 11, № 4, 361-374 (1975).

13. Rom a n, J. R., Bullo ck, T. E., IEEE Trans. Automat. Contr., 20, № 4, 529-533 (1975)

14. Кв акерна а к X., С и в ан Р., Линейные оптимальные системы управления, М., «Мир», 1977.

15. Porter, B., Crossley, R., Modal control, Taylor and Francis, London, 1972.

Ннститут кибернетики

Академии наук Эстонской ССР

Поступила в редакцию 20/II 1980

\section{O. NURGES}

\section{MITMEMOOTMELISE LINEAARSE SUSTEEMI KANOONILISTE KUJUDE VORDLEV ANALUUS}

Artiklis on vaadeldud mitmemõõtmelise dünaamilise süsteemi ekvivalentseid esitusi Luenbergeri, Budini ja Jordani kanoonilisel kujul, kusjuures põhitähelepanu on pööratud tundmatute arvu minimeerimisele ja nende asukoha fikseerimisele. 


\section{U. NURGES}

\section{THE COMPARATIVE ANALYSIS OF THE CANONICAL FORMS OF MULTIVARIABLE LINEAR SYSTEMS}

Luenberger's, Budin's and Jordan's canonical forms of the $n$-th order linear dynamical system with $m$ inputs and $p$ outputs are considered. The main aim of the paper is to minimize the amount of unknown parameters in these canonical forms. The formulae are given for determining the number of unknown parameters from structural invariants of the canonical form, as well as the inequalities for fixing the location of unknown parameters. A classification of canonical forms of the linear dynamical system is presented.

Of special interest is the class of Luenberger's canonical forms. All of the most popular observable canonical forms (c. f. of Bucy, row-companion c. f., observable c. f.) are considered and their place in the family of Luenberger's canonical forms is pointed out.

Besides the classification and comparison of the well-known canonical forms, the paper contains some new results conserned with the second canonical form of Luenberger. The considerable amount of parameters of matrices $A$ and $C$ are fixed in addition to the structurally fixed parameters. This way it was possible to show that the second canonical form has as many unknown parameters as the first one. Since the matrix $C$ has not in the first c. f. any unknown parameter, so has the matrix $A$ in the second c. f. fewer unknown parameters than in the first c. f.

The same results can be obtained for the matrices $A$ and $B$ in controllable canonicai forms by using the duality relations. 